\title{
Some New Results on Fixed Points of Meromorphic Functions Defined in Annuli
}

\author{
Zhaojun Wu, ${ }^{1}$ Zuxing Xuan, ${ }^{2}$ and Yuxian Chen ${ }^{3}$ \\ ${ }^{1}$ School of Mathematics and Statistics, Hubei University of Science and Technology, Xianning 437100, China \\ ${ }^{2}$ Beijing Key Laboratory of Information Service Engineering, Department of General Education, Beijing Union University, \\ No. 97 Bei Si Huan Dong Road, Chaoyang District, Beijing 100101, China \\ ${ }^{3}$ School of Mathematics and Computer Science, Xinyu University, Xinyu 338004, China
}

Correspondence should be addressed to Zuxing Xuan; zuxingxuan@163.com

Received 16 July 2015; Accepted 9 November 2015

Academic Editor: Adrian Petrusel

Copyright (C) 2015 Zhaojun Wu et al. This is an open access article distributed under the Creative Commons Attribution License, which permits unrestricted use, distribution, and reproduction in any medium, provided the original work is properly cited.

The purpose of this paper is to investigate the fixed points of meromorphic functions in annuli. Some well-known facts of fixed points for meromorphic functions in the plane will be considered in annuli.

\section{Introduction and Main Results}

Let $f(z)$ be a meromorphic function. A point $z$ is called a fixed point if $f(z)=z$. There are a considerable number of results on the fixed points for meromorphic functions in the plane; we refer the reader to Chuang and Yang [1]. In 1988, Zhu [2] has proved the following.

Theorem A. Let $f$ be a transcendental meromorphic function in the plane. Then either $f$ or $f^{\prime}$ has infinitely many fixed points.

In the 1960s, Baker [3] proved that if $f(z)$ is a transcendental entire function in the plane such that there exists $a \in \mathbb{C}$ with $\delta(a, f)>0$, then $f$ has fixed points of order one. In 1993, Lahiri [4] gave an extension of Baker's results and proved the following theorem.

Theorem B. Let $f$ be a transcendental meromorphic function in the plane. Suppose that there exists $a \in \mathbb{C}$ with $\delta(a, f)>0$ and $N(r, f)=S(r, f)$. Then $f$ has infinitely many fixed points.

In recent ten years some well-known facts of the value distribution theory for meromorphic function in the plane were extended for the meromorphic function in the annuli:

$$
A\left(R_{0}\right)=\left\{z: \frac{1}{R_{0}}<|z|<R_{0}\right\}, \quad 1<R_{0} \leq+\infty .
$$

In 2005, Khrystiyanyn and Kondratyuk [5, 6] gave an extension of the Nevanlinna value distribution theory for meromorphic functions in annuli. In their extension the main characteristics of meromorphic functions are one-parameter and possess the same properties as in the classical case of a simply connected domain. In [5, 6], we can get the analogues of Jensen's formula, the first fundamental theorem, the lemma on logarithmic derivative, and the second fundamental theorem of the Nevanlinna theory for meromorphic functions in annuli. Lund and $Y e$ [7] investigated logarithmic derivatives in the annuli $\{z$ : $\left.R_{1}<|z|<R_{2}\right\}$. After [5, 6], Cao et al. [8-11] study the uniqueness of the meromorphic functions in annuli, Chen and $W u$ [12] study the exceptional values for meromorphic function and its derivatives in annuli, and Fernández [13] studies the value distribution of meromorphic functions in the punctured plane $A(\infty)$. The main purpose of this paper is to study the fixed points of meromorphic functions in annuli. We will prove the following theorems by using the similar method as in $[2,4]$.

Theorem 1. Let $f$ be an admissible meromorphic function in $A\left(R_{0}\right), 1<R_{0} \leq+\infty$. Then either $f$ or $f^{\prime}$ has infinitely many fixed points.

Theorem 2. Let $f$ be an admissible meromorphic function in $A\left(R_{0}\right), 1<R_{0} \leq+\infty$. Suppose that there exists $a \in \mathbb{C}$ with 
$\delta_{0}(a, f)>0$ and $N_{0}(R, f)=S(R, f)$. Then both $f$ and $f^{\prime}$ have infinitely many fixed points.

\section{Nevanlinna Theory in Annuli}

In the following, we introduce the definitions, notations, and results of $[5,6,8,14]$ which will be used in this paper. Let $f(z)$ be a meromorphic function in $A\left(R_{0}\right)$. Denote

$$
\begin{gathered}
m\left(R, \frac{1}{f-a}\right)=\frac{1}{2 \pi} \int_{0}^{2 \pi} \log ^{+} \frac{1}{\left|f\left(R e^{i \theta}\right)-a\right|} d \theta, \\
m(R, f)=\frac{1}{2 \pi} \int_{0}^{2 \pi} \log ^{+}\left|f\left(R e^{i \theta}\right)\right| d \theta,
\end{gathered}
$$

where $a \in \mathbb{C}$ and $1 / R_{0}<R<R_{0}$. Let

$$
\begin{aligned}
m_{0}\left(R, \frac{1}{f-a}\right)= & m\left(R, \frac{1}{f-a}\right)+m\left(\frac{1}{R}, \frac{1}{f-a}\right) \\
& -2 m\left(1, \frac{1}{f-a}\right), \quad 1<R<R_{0}, \\
m_{0}(R, f)= & m(R, f)+m\left(\frac{1}{R}, f\right)-2 m(1, f), \\
& 1<R<R_{0} .
\end{aligned}
$$

Put

$$
\begin{aligned}
& N_{1}\left(R, \frac{1}{f-a}\right)=\int_{1 / R}^{1} \frac{n_{1}(t, 1 /(f-a))}{t} d t, \\
& N_{2}\left(R, \frac{1}{f-a}\right)=\int_{1}^{R} \frac{n_{2}(t, 1 /(f-a))}{t} d t,
\end{aligned}
$$

where $1<R<R_{0}, n_{1}(t, 1 /(f-a))$ is the counting function of poles of the function $1 /(f-a)$ in $\{z: t<|z| \leq 1\}$, and $n_{2}(t, 1 /(f-a))$ is the counting function of poles of the function $1 /(f-a)$ in $\{z: 1<|z| \leq t\}$. Denote also

$$
\begin{aligned}
& N_{1}(R, f)=\int_{1 / R}^{1} \frac{n_{1}(t, f)}{t} d t, \\
& N_{2}(R, f)=\int_{1}^{R} \frac{n_{2}(t, f)}{t} d t,
\end{aligned}
$$

where $1<R<R_{0}, n_{1}(t, f)$ is the counting function of poles of the function $f$ in $\{z: t<|z| \leq 1\}$, and $n_{2}(t, f)$ is the counting function of poles of the function $f$ in $\{z: 1<|z| \leq t\}$. Let

$$
\begin{aligned}
N_{0}\left(R, \frac{1}{f-a}\right) & =N_{1}\left(R, \frac{1}{f-a}\right)+N_{2}\left(R, \frac{1}{f-a}\right), \\
N_{0}(R, f) & =N_{1}(R, f)+N_{2}(R, f) .
\end{aligned}
$$

Finally, we define the Nevanlinna characteristic of $f$ in $A\left(R_{0}\right)$ by

$$
T_{0}(R, f)=m_{0}(R, f)+N_{0}(R, f), \quad 1<R<R_{0},
$$

where $R_{0} \leq+\infty$. Suppose that $f, g$ are two meromorphic functions in $A\left(R_{0}\right)$, where $1<R_{0} \leq+\infty$. Then

$$
\begin{aligned}
& T_{0}(R, f)=T_{0}\left(R, \frac{1}{f}\right), \\
& \max \left\{m_{0}(R, f g), m_{0}\left(R, \frac{f}{g}\right), m_{0}(R, f \pm g)\right\} \\
& \quad \leq m_{0}(R, f)+m_{0}(R, g)+O(1), \\
& \max \left\{T_{0}(R, f g), T_{0}\left(R, \frac{f}{g}\right), T_{0}(R, f \pm g)\right\} \\
& \quad \leq T_{0}(R, f)+T_{0}(R, g)+O(1) .
\end{aligned}
$$

Definition 3. Let $f$ be a nonconstant meromorphic function in $A\left(R_{0}\right)$. One calls $f$ admissible if

$$
\limsup _{R \rightarrow+\infty} \frac{T_{0}(R, f)}{\log R}=\infty, \quad 1 \leq R<R_{0}=+\infty,
$$

or

$$
\limsup _{R \rightarrow R_{0}} \frac{T_{0}(R, f)}{-\log \left(R_{0}-R\right)}=\infty, \quad 1 \leq R<R_{0}=+\infty .
$$

Definition 4. Let $f$ be a nonconstant meromorphic function in $A\left(R_{0}\right), 1<R_{0} \leq+\infty$, and $a \in \mathbb{C}$. Then the value

$$
\delta_{0}(a, f)=1-\limsup _{R \rightarrow R_{0}} \frac{N_{0}(R, 1 /(f-a))}{T_{0}(R, f)}
$$

is called the deficiency of the function $f$ for the value $a$. For $a=\infty$, one sets

$$
\delta_{0}(\infty, f)=1-\limsup _{R \rightarrow R_{0}} \frac{N_{0}(R, f)}{T_{0}(R, f)} .
$$

Throughout, we denote by $S(R, *)$ quantities that satisfy the following:

(1) for the case $R_{0}=+\infty$,

$$
S(R, *)=O\left(\log \left(R T_{0}(R, f)\right)\right),
$$

for $R \in(1,+\infty)$ except for the set $\Delta_{R}$ such that $\int_{\Delta_{R}} R^{\lambda-1} d R<+\infty$;

(2) for the case $R_{0}<+\infty$,

$$
S(R, *)=O\left(\log \left(\frac{T_{0}(R, f)}{R_{0}-R}\right)\right),
$$

for $R \in\left(1, R_{0}\right)$ except for the set $\Delta_{R}^{\prime}$ such that $\int_{\Delta_{R}^{\prime}}\left(d R /\left(R_{0}-R\right)^{\lambda-1}\right)<+\infty$.

Thus, for an admissible meromorphic function in $A\left(R_{0}\right), 1<$ $R_{0} \leq+\infty, S(R, f)=O\left(T_{0}(R, f)\right)$ holds for all $1 \leq R<R_{0} \leq$ $+\infty$ except for the set $\Delta_{R}$ or the set $\Delta_{R}^{\prime}$ mentioned above, respectively. Under the above notations, we give the following theorems which will be used in the proof of Theorems 1 and 2 . 
Theorem C (the first fundamental theorem). Let $f$ be $a$ nonconstant meromorphic function in $A\left(R_{0}\right)$, where $1<R_{0} \leq$ $+\infty$. Then

$$
T_{0}\left(R, \frac{1}{f-a}\right)=T_{0}(R, f)+O(1), \quad 1<R<R_{0},
$$

for every fixed $a \in \mathbb{C}$.

Theorem D (lemma on the logarithmic derivative). Let $f$ be an admissible meromorphic function in $A\left(R_{0}\right), 1<R_{0} \leq+\infty$. Then $m_{0}\left(R, f^{(k)} / f\right)=S(R, f)$ holds for every positive integer $k$.

\section{Proof of Theorem 1}

Lemma 5. Let $f$ be an admissible meromorphic function in $A\left(R_{0}\right), 1<R_{0} \leq+\infty$. Then

$$
\begin{aligned}
T_{0}(R, f) \leq & \bar{N}_{0}(R, f)+N_{0}\left(R, \frac{1}{f}\right) \\
& +N_{0}\left(R, \frac{1}{f^{\prime}-z+1}\right)+S(R, f) .
\end{aligned}
$$

Proof. Consider

$$
\frac{1}{f}=\frac{f^{\prime}}{\varphi f}-\frac{\varphi f^{\prime \prime}-f^{\prime}}{\varphi f} \frac{f^{\prime}-\varphi}{\varphi f^{\prime \prime}-f^{\prime}},
$$

where $\varphi=\varphi(z)=z-1$. This leads to

$$
\begin{aligned}
m_{0}\left(R, \frac{1}{f}\right) \leq & m_{0}\left(R, \frac{f^{\prime}}{\varphi f}\right)+m_{0}\left(R, \frac{\varphi f^{\prime \prime}-f^{\prime}}{\varphi f}\right) \\
& +m_{0}\left(R, \frac{f^{\prime}-\varphi}{\varphi f^{\prime \prime}-f^{\prime}}\right) .
\end{aligned}
$$

Applying the first fundamental theorem, we get

$$
\begin{aligned}
m_{0}\left(R, \frac{1}{f}\right)= & T_{0}(R, f)-N_{0}\left(R, \frac{1}{f}\right) \\
& +O(1), \\
m_{0}\left(R, \frac{f^{\prime}-\varphi}{\varphi f^{\prime \prime}-f^{\prime}}\right)= & m_{0}\left(R, \frac{\varphi f^{\prime \prime}-f^{\prime}}{f^{\prime}-\varphi}\right) \\
& +N_{0}\left(R, \frac{\varphi f^{\prime \prime}-f^{\prime}}{f^{\prime}-\varphi}\right) \\
& -N_{0}\left(R, \frac{f^{\prime}-\varphi}{\varphi f^{\prime \prime}-f^{\prime}}\right)+O(1) \\
= & m_{0}\left(R, \frac{\varphi f^{\prime \prime}-f^{\prime}}{f^{\prime}-\varphi}\right) \\
& +N_{0}\left(R, \frac{1}{f^{\prime}-\varphi}\right) \\
& +N_{0}\left(R, \varphi f^{\prime \prime}-f^{\prime}\right)
\end{aligned}
$$

$$
\begin{aligned}
& -N_{0}\left(R, f^{\prime}-\varphi\right) \\
& -N_{0}\left(R, \frac{1}{\varphi f^{\prime \prime}-f^{\prime}}\right)+O(1) \\
\leq & m_{0}\left(R, \frac{\varphi f^{\prime \prime}-f^{\prime}}{f^{\prime}-\varphi}\right) \\
& +N_{0}\left(R, \frac{1}{f^{\prime}-\varphi}\right)+\bar{N}_{0}(R, f) \\
& -N_{0}\left(R, \frac{1}{\varphi f^{\prime \prime}-f^{\prime}}\right)+O(1) .
\end{aligned}
$$

Substituting (21) into (20), noting that $m_{0}(R, \varphi)=S(R, f)$, $S\left(R, f^{\prime}-\varphi\right)=S(R, f)$, we have

$$
\begin{aligned}
& T_{0}(R, f) \leq N_{0}\left(R, \frac{1}{f}\right)+2 m_{0}\left(R, \frac{f^{\prime}}{\varphi f}\right) \\
& +m_{0}\left(R, \frac{f^{\prime}}{f}\right)+m_{0}\left(R, \frac{\varphi f^{\prime \prime}-f^{\prime}}{f^{\prime}-\varphi}\right) \\
& +N_{0}\left(R, \frac{1}{f^{\prime}-\varphi}\right)+\bar{N}_{0}(R, f) \\
& -N_{0}\left(R, \frac{1}{\varphi f^{\prime \prime}-f^{\prime}}\right)+O(1) \\
& \leq N_{0}\left(R, \frac{1}{f}\right)+2 m_{0}\left(R, \frac{f^{\prime}}{\varphi f}\right) \\
& +m_{0}\left(R, \frac{f^{\prime}}{f}\right) \\
& +m_{0}\left(R, \frac{\left(f^{\prime}-\varphi\right)^{\prime}-\left(f^{\prime}-\varphi\right)}{f^{\prime}-\varphi}\right) \\
& +N_{0}\left(R, \frac{1}{f^{\prime}-\varphi}\right)+\bar{N}_{0}(R, f) \\
& -N_{0}\left(R, \frac{1}{\varphi f^{\prime \prime}-f^{\prime}}\right)+O(1) \\
& =\bar{N}_{0}(R, f)+N_{0}\left(R, \frac{1}{f}\right) \\
& +N_{0}\left(R, \frac{1}{f^{\prime}-\varphi}\right)-N_{0}\left(R, \frac{1}{\varphi f^{\prime \prime}-f^{\prime}}\right) \\
& +S(R, f) \text {. }
\end{aligned}
$$

Lemma 6. Suppose that $f$ is an admissible meromorphic function in $A\left(R_{0}\right), 1<R_{0} \leq+\infty$. Then

$$
\begin{aligned}
N_{0}^{1)}(R, f) \leq & 9 N_{0}\left(R, \frac{1}{f}\right)+9 N_{0}\left(R, \frac{1}{f^{\prime}-z+1}\right) \\
& +S(R, f),
\end{aligned}
$$


where $N_{0}^{1)}(R, f)$ is the $N_{0}$ function of $n^{1)}(t, f)$ which denote the number of simple poles of $f$.

Proof. We define

$$
\begin{aligned}
& P(z)=\frac{f\left(f^{\prime}-\varphi\right)}{\varphi f^{\prime \prime}-f^{\prime}} \\
& G(z)=(P \varphi)^{\prime \prime}+\left(P \varphi^{\prime}\right)^{\prime}+\varphi=3 P^{\prime}+\varphi P^{\prime \prime}+\varphi,
\end{aligned}
$$

where $\varphi=\varphi(z)=z-1$. Let $z_{0} \in A\left(R_{0}\right)$ be a simple pole of $f(z)$. Then as in [2] we have $G\left(z_{0}\right)=0$. Therefore, when $G(z) \neq \equiv$, by the first fundamental theorem and (10), noting that $T_{0}(R, \varphi)=S(R, f), S(R, P)=S(R, f)$, we have

$$
\begin{aligned}
N_{0}^{1)}(R, f) \leq & N_{0}\left(R, \frac{1}{G}\right) \leq T_{0}\left(R, \frac{1}{G}\right) \\
= & T_{0}(R, G)+O(1) \\
= & T_{0}\left(R, P\left(\frac{3 P^{\prime}}{P}+\frac{\varphi P^{\prime \prime}}{P}\right)+\varphi\right)+O(1) \\
\leq & T_{0}(R, P)+T_{0}\left(R, P^{\prime}\right)+T_{0}\left(R, P^{\prime \prime}\right) \\
& +2 T_{0}\left(R, \frac{1}{P}\right)+S(R, f) \\
\leq & 7 T_{0}(R, P)+2 T_{0}\left(R, \frac{1}{P}\right)+S(R, f) \\
& +S(R, P) \leq 9 T_{0}\left(R, \frac{1}{P}\right)+S(R, f) .
\end{aligned}
$$

Now we estimate $T_{0}(R, 1 / P)$. Firstly, we have

$$
\begin{aligned}
m_{0}\left(R, \frac{1}{P}\right)= & m_{0}\left(R, \frac{\varphi f^{\prime \prime}-f^{\prime}}{f\left(f^{\prime}-\varphi\right)}\right) \\
\leq & m_{0}\left(R, \frac{\left(\left(f^{\prime}-\varphi\right) / f^{\prime}\right)^{\prime}}{\left(f^{\prime}-\varphi\right) / f^{\prime}}\right) \\
& +m_{0}\left(R, \frac{f^{\prime}}{f}\right)=S(R, f) .
\end{aligned}
$$

In [2], Zhu indicated that poles of $1 / P$ can occur only at zeros of $f(z)$ or $f^{\prime}-\varphi$. But every zero with multiplicity $k$ of $f^{\prime}-\varphi$ gives a pole of $1 / P$ once at most, for it must be the zero of $\varphi f^{\prime \prime}-f^{\prime}$ with multiplicity being at least $k-1$. So we have

$$
N_{0}\left(R, \frac{1}{P}\right) \leq N_{0}\left(R, \frac{1}{f}\right)+N_{0}\left(R, \frac{1}{f^{\prime}-\varphi}\right) .
$$

From (25), (26), and (27) we derive

$$
\begin{aligned}
N_{0}^{1)}(R, f) \leq & 9 N_{0}\left(R, \frac{1}{f}\right)+9 N_{0}\left(R, \frac{1}{f^{\prime}-\varphi}\right) \\
& +S(R, f) .
\end{aligned}
$$

By a similar discussion as in [2], one can prove Lemma 6 in the case of $G(z) \equiv 0$.

We are now in the position to prove Theorem 1.

Proof. Note $\bar{N}_{0}(R, f)=N_{0}^{1)}(R, f)+\bar{N}_{0}^{(2}(R, f)$, where $\bar{N}_{0}^{(2}$ is the $N_{0}$ function of $\bar{n}^{(2}(t, f)$ which denote the number of multiple poles of $f$, ignoring multiplicity. By (18) we have

$$
\begin{aligned}
2 \bar{N}_{0}^{(2}(R, f)+N_{0}^{1)}(R, f) \leq N_{0}(R, f) \leq T_{0}(R, f) & \leq N_{0}^{1)}(R, f)+\bar{N}_{0}^{(2}(R, f)+N_{0}\left(R, \frac{1}{f}\right) \\
& +N_{0}\left(R, \frac{1}{f^{\prime}-z+1}\right)+S(R, f) .
\end{aligned}
$$

Thus,

$$
\begin{aligned}
\bar{N}_{0}^{(2}(R, f) \leq & N_{0}\left(R, \frac{1}{f}\right)+N_{0}\left(R, \frac{1}{f^{\prime}-z+1}\right) \\
& +S(R, f) .
\end{aligned}
$$

By (18), (23), and (30) we have

$$
\begin{aligned}
T_{0}(R, f) \leq & N_{0}^{1)}(R, f)+\bar{N}_{0}^{(2}(R, f)+N_{0}\left(R, \frac{1}{f}\right) \\
& +N_{0}\left(R, \frac{1}{f^{\prime}-z+1}\right)+S(R, f) \\
\leq & 11 N_{0}\left(R, \frac{1}{f}\right)+11 N_{0}\left(R, \frac{1}{f^{\prime}-z+1}\right) \\
& +S(R, f) .
\end{aligned}
$$

Denoting $g \equiv f-z$, by (31) we derive

$$
\begin{aligned}
T_{0}(R, f) \leq & T_{0}(R, g)+O(1) \\
\leq & 11 N_{0}\left(R, \frac{1}{g}\right)+11 N_{0}\left(R, \frac{1}{g^{\prime}-z+1}\right) \\
& +S(R, g)+O(1) \\
\leq & 11 N_{0}\left(R, \frac{1}{f-z}\right)+11 N_{0}\left(R, \frac{1}{f^{\prime}-z}\right) \\
& +S(R, f) .
\end{aligned}
$$

If both $f$ and $f^{\prime}$ have only a finite number of fixed points, then from (32) we would have

$$
T_{0}(R, f) \leq S(R, f) .
$$

This leads to a contradiction and Theorem 1 is proved. 


\section{Proof of Theorem 2}

Proof. Consider

$$
\frac{1}{f}=\frac{f^{\prime}}{z f}-\frac{z f^{\prime \prime}-f^{\prime}}{z f} \frac{f^{\prime}-z}{z f^{\prime \prime}-f^{\prime}} .
$$

This leads to

$$
\begin{aligned}
m_{0}\left(R, \frac{1}{f}\right) \leq & m_{0}\left(R, \frac{f^{\prime}}{z f}\right)+m_{0}\left(R, \frac{z f^{\prime \prime}-f^{\prime}}{z f}\right) \\
& +m_{0}\left(R, \frac{f^{\prime}-z}{z f^{\prime \prime}-f^{\prime}}\right) .
\end{aligned}
$$

Applying the first fundamental theorem, we get

$$
\begin{aligned}
& m_{0}\left(R, \frac{1}{f}\right)=T_{0}(R, f)-N_{0}\left(R, \frac{1}{f}\right) \\
& +O(1) \text {, } \\
& m_{0}\left(R, \frac{f^{\prime}-z}{z f^{\prime \prime}-f^{\prime}}\right)=m_{0}\left(R, \frac{z f^{\prime \prime}-f^{\prime}}{f^{\prime}-z}\right) \\
& +N_{0}\left(R, \frac{z f^{\prime \prime}-f^{\prime}}{f^{\prime}-z}\right) \\
& -N_{0}\left(R, \frac{f^{\prime}-z}{z f^{\prime \prime}-f^{\prime}}\right)+O(1) \\
& =m_{0}\left(R, \frac{z f^{\prime \prime}-f^{\prime}}{f^{\prime}-z}\right) \\
& +N_{0}\left(R, \frac{1}{f^{\prime}-z}\right) \\
& +N_{0}\left(R, z f^{\prime \prime}-f^{\prime}\right) \\
& -N_{0}\left(R, f^{\prime}-z\right) \\
& -N_{0}\left(R, \frac{1}{z f^{\prime \prime}-f^{\prime}}\right)+O(1) \\
& \leq m_{0}\left(R, \frac{z f^{\prime \prime}-f^{\prime}}{f^{\prime}-z}\right) \\
& +N_{0}\left(R, \frac{1}{f^{\prime}-z}\right)+\bar{N}_{0}(R, f) \\
& -N_{0}\left(R, \frac{1}{z f^{\prime \prime}-f^{\prime}}\right)+O(1) .
\end{aligned}
$$

Substituting (36) into (35), we have

$$
\begin{aligned}
T_{0}(R, f) \leq & N_{0}\left(R, \frac{1}{f}\right)+2 m_{0}\left(R, \frac{f^{\prime}}{z f}\right) \\
& +m_{0}\left(R, \frac{f^{\prime}}{f}\right)+m_{0}\left(R, \frac{z f^{\prime \prime}-f^{\prime}}{f^{\prime}-z}\right) \\
& +N_{0}\left(R, \frac{1}{f^{\prime}-z}\right)+\bar{N}_{0}(R, f)
\end{aligned}
$$

$$
-N_{0}\left(R, \frac{1}{z f^{\prime \prime}-f^{\prime}}\right)+O(1)
$$$$
\leq N_{0}\left(R, \frac{1}{f}\right)+2 m_{0}\left(R, \frac{f^{\prime}}{z f}\right)
$$$$
+m_{0}\left(R, \frac{f^{\prime}}{f}\right)
$$$$
+m_{0}\left(R, \frac{\left(f^{\prime}-z\right)^{\prime}-\left(f^{\prime}-z\right)}{f^{\prime}-z}\right)
$$$$
+N_{0}\left(R, \frac{1}{f^{\prime}-z}\right)+\bar{N}_{0}(R, f)
$$$$
-N_{0}\left(R, \frac{1}{z f^{\prime \prime}-f^{\prime}}\right)+O(1)
$$$$
\leq \bar{N}_{0}(R, f)+N_{0}\left(R, \frac{1}{f}\right)
$$$$
+N_{0}\left(R, \frac{1}{f^{\prime}-z}\right)+S(R, f) \text {. }
$$

Denoting $g \equiv f-a$, by (37) we derive

$$
\begin{aligned}
T_{0}(R, f) \leq & T_{0}(R, g)+O(1) \\
\leq & \bar{N}_{0}(R, g)+N_{0}\left(R, \frac{1}{g}\right) \\
& +N_{0}\left(R, \frac{1}{g^{\prime}-z}\right)+S(R, g) \\
\leq & N_{0}(R, f)+N_{0}\left(R, \frac{1}{f-a}\right) \\
& +N_{0}\left(R, \frac{1}{f^{\prime}-z}\right)+S(R, f) .
\end{aligned}
$$

Since $N_{0}(R, f)=S(R, f)$, by (38), we can get

$$
\begin{aligned}
T_{0}(R, f) \leq & N_{0}\left(R, \frac{1}{f-a}\right)+N_{0}\left(R, \frac{1}{f^{\prime}-z}\right) \\
& +S(R, f) .
\end{aligned}
$$

Since $\delta_{0}(a, f)>0$, then there is a positive number $\theta<1$ such that

$$
N_{0}\left(R, \frac{1}{f-a}\right)<\theta T_{0}(R, f) .
$$

If $f^{\prime}$ have only a finite number of fixed points, then from (39) and (40) we would have

$$
(1-\theta) T_{0}(R, f) \leq S(R, f) .
$$

This leads to a contradiction and $f^{\prime}$ has infinitely many fixed points. In the following, we will prove that $f$ also has infinitely many fixed points under the condition of Theorem 2 . 
If $a^{[1]}, a^{[2]}, \ldots, a^{[p]}$ are distinct finite complex numbers, put

$$
F(z)=\sum_{i=1}^{p} \frac{1}{f(z)-a^{[i]}} .
$$

Then as in [15] we have

$$
\begin{gathered}
m(R, F) \geq \sum_{i=1}^{p} m\left(R, \frac{1}{f(z)-a^{[i]}}\right)+O(1), \\
m\left(\frac{1}{R}, F\right) \geq \sum_{i=1}^{p} m\left(\frac{1}{R}, \frac{1}{f(z)-a^{[i]}}\right)+O(1) .
\end{gathered}
$$

Since

$$
\begin{aligned}
m(R, F) & \leq m\left(R, f^{\prime} F\right)+m\left(R, \frac{1}{f^{\prime}}\right) \\
& \leq \sum_{i=1}^{p} m\left(R, \frac{f^{\prime}}{f-a^{[i]}}\right)+m\left(R, \frac{1}{f^{\prime}}\right), \\
m\left(\frac{1}{R}, F\right) & \leq m\left(\frac{1}{R}, f^{\prime} F\right)+m\left(\frac{1}{R}, \frac{1}{f^{\prime}}\right) \\
& \leq \sum_{i=1}^{p} m\left(\frac{1}{R}, \frac{f^{\prime}}{f-a^{[i]}}\right)+m\left(\frac{1}{R}, \frac{1}{f^{\prime}}\right),
\end{aligned}
$$

therefore,

$$
\begin{aligned}
m_{0}(R, F) \leq & \sum_{i=1}^{p} m_{0}\left(R, \frac{f^{\prime}}{f-a^{[i]}}\right)+m_{0}\left(R, \frac{1}{f^{\prime}}\right) \\
& +O(1) .
\end{aligned}
$$

It follows from (43)-(45) and Theorem D that

$$
\sum_{i=1}^{p} m_{0}\left(R, \frac{1}{f(z)-a^{[i]}}\right) \leq m_{0}\left(R, \frac{1}{f^{\prime}}\right)+S(R, f) .
$$

In particular, we have

$$
m_{0}\left(R, \frac{1}{f(z)-a}\right) \leq m_{0}\left(R, \frac{1}{f^{\prime}}\right)+S(R, f) .
$$

Applying (46) to the function $f-z$ we obtain

$$
m_{0}\left(R, \frac{1}{f(z)-z}\right) \leq m_{0}\left(R, \frac{1}{f^{\prime}-1}\right)+S(R, f) .
$$

Further application of (46) gives

$$
\begin{gathered}
m_{0}\left(R, \frac{1}{f^{\prime}}\right)+m_{0}\left(R, \frac{1}{f^{\prime}-1}\right) \\
\quad \leq m_{0}\left(R, \frac{1}{f^{\prime \prime}}\right)+S\left(R, f^{\prime}\right) \\
\quad \leq T_{0}\left(R, f^{\prime \prime}\right)+S(R, f) .
\end{gathered}
$$

On the other hand, in view of Theorems C and D, one has

$$
\begin{aligned}
T_{0}\left(R, f^{\prime \prime}\right) & =m_{0}\left(R, f^{\prime \prime}\right)+N_{0}\left(R, f^{\prime \prime}\right) \\
& \leq m_{0}(R, f)+m_{0}\left(R, \frac{f^{\prime \prime}}{f}\right)+N_{0}\left(R, f^{\prime \prime}\right) \\
& =m_{0}(R, f)+N_{0}\left(R, f^{\prime \prime}\right)+S(R, f) .
\end{aligned}
$$

If $z_{0}$ is a pole of order $k$, it is a pole of $f^{\prime \prime}$ or order $k+2 \leq$ $3 k$. So $N_{0}\left(R, f^{\prime \prime}\right) \leq 3 N_{0}(R, f)$ and by the given condition $N_{0}\left(R, f^{\prime \prime}\right) \leq S(R, f)$. Therefore, we get from (50) that

$$
T_{0}\left(R, f^{\prime \prime}\right) \leq T_{0}(R, f)+S(R, f) .
$$

Combining (47), (48), and (49) with (51), we have

$$
\begin{aligned}
& m_{0}\left(R, \frac{1}{f(z)-a}\right)+m_{0}\left(R, \frac{1}{f(z)-z}\right) \\
& \quad \leq T_{0}(R, f)+S(R, f) .
\end{aligned}
$$

By Theorem C and (10), we see that

$$
\begin{aligned}
T_{0}(R, f) \leq & N_{0}\left(R, \frac{1}{f(z)-a}\right)+N_{0}\left(R, \frac{1}{f(z)-z}\right) \\
& +S(R, f) .
\end{aligned}
$$

If $f$ has only a finite number of fixed points, then from (40) and (53) we would have

$$
(1-\theta) T_{0}(R, f) \leq S(R, f) .
$$

This leads to a contradiction and $f$ has infinitely many fixed points.

\section{Conflict of Interests}

The authors declare that there is no conflict of interests regarding the publication of this paper.

\section{Acknowledgments}

Zuxing Xuan is the corresponding author and he is supported in part by NNSFC (no. 91420202) and the Project of Construction of Innovative Teams and Teacher Career Development for Universities and Colleges under Beijing Municipality (CIT and TCD201504041, IDHT20140508). This research was partly supported by the National Natural Science Foundation of China (Grant no. 11201395) and by the Science Foundation of Educational Commission of Hubei Province (Grant no. Q20132801). Yuxian Chen is supported in part by University Science and Technology Project of Jiangxi Province (Grant no. GJJ14774).

\section{References}

[1] C. T. Chuang and C. C. Yang, Theory of Fix Points and Factorization of Meromorphic Functions, Mathematical Monogragh Series, Peking University Press, 1986. 
[2] J. H. Zhu, “The general form of Hayman's inequality and the fixed points of meromorphic functions," Kexue Tongbao, vol. 33, no. 4, pp. 265-269, 1988.

[3] I. N. Baker, "Some entire functions with fix-points of every order," Journal of the Australian Mathematical Society, vol. 1, pp. 203-209, 1960.

[4] I. Lahiri, "Milloux theorem, deficiency and fix-points for vectorvalued meromorphic functions," The Journal of the Indian Mathematical Society, vol. 59, no. 1-4, pp. 45-60, 1993.

[5] A. Y. Khrystiyanyn and A. A. Kondratyuk, "On the Nevanlinna theory for meromorphic functions on annuli. I," Mathematychni Studii, vol. 23, pp. 19-30, 2005.

[6] A. Y. Khrystiyanyn and A. A. Kondratyuk, "On the Nevanlinna theory for meromorphic functions on annuli II," Mathematychni Studii, vol. 24, no. 2, pp. 57-68, 2005.

[7] M. E. Lund and Z. Ye, "Logarithmic derivatives in annuli," Journal of Mathematical Analysis and Applications, vol. 356, no. 2, pp. 441-452, 2009.

[8] T.-B. Cao and Z.-S. Deng, "On the uniqueness of meromorphic functions that share three or two finite sets on annuli," Proceedings-Mathematical Sciences, vol. 122, no. 2, pp. 203220, 2012.

[9] T. B. Cao and Y. H. Yi, "Uniqueness theorems of meromorphic functions shares sets IM on Annuli," Acta Mathematica Sinica (Chinese Series), vol. 54, pp. 623-632, 2011.

[10] T.-B. Cao, H.-X. Yi, and H.-Y. Xu, "On the multiple values and uniqueness of meromorphic functions on annuli," Computers \& Mathematics with Applications, vol. 58, no. 7, pp. 1457-1465, 2009.

[11] H.-Y. Xu and Z.-X. Xuan, "The uniqueness of analytic functions on annuli sharing some values," Abstract and Applied Analysis, vol. 2012, Article ID 896596, 13 pages, 2012.

[12] Y. X. Chen and Z. J. Wu, "Exceptional values of meromorphic functions and of their derivatives on annuli," Annales Polonici Mathematici, vol. 105, no. 2, pp. 154-165, 2012.

[13] A. Fernández, "On the value distribution of meromorphic function in the punctured plane," Mathematychni Studii, vol. 34, pp. 136-144, 2010.

[14] A. A. Kondratyuk and I. Laine, "Meromorphic functions in multiply connected domains," in Fourier Series Methods in Complex Analysis. Proceedings of the Workshop, Mekrijärvi, Finland, July 2005, vol. 10, pp. 9-111, University of Joensuu, Department of Mathematics, 2006.

[15] L. Yang, Value Distribution Theory, Translated and Revised from the 1982 Chinese Original, Springer, Berlin, Germany; Science Press, Beijing, China, 1993. 


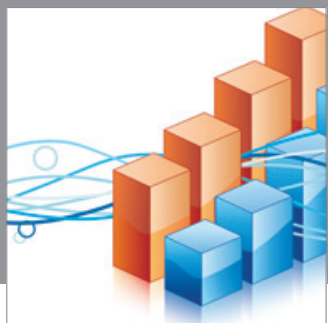

Advances in

Operations Research

mansans

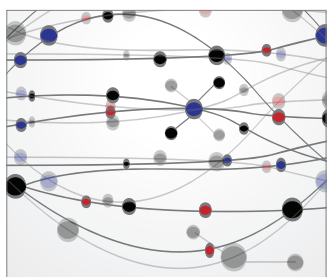

The Scientific World Journal
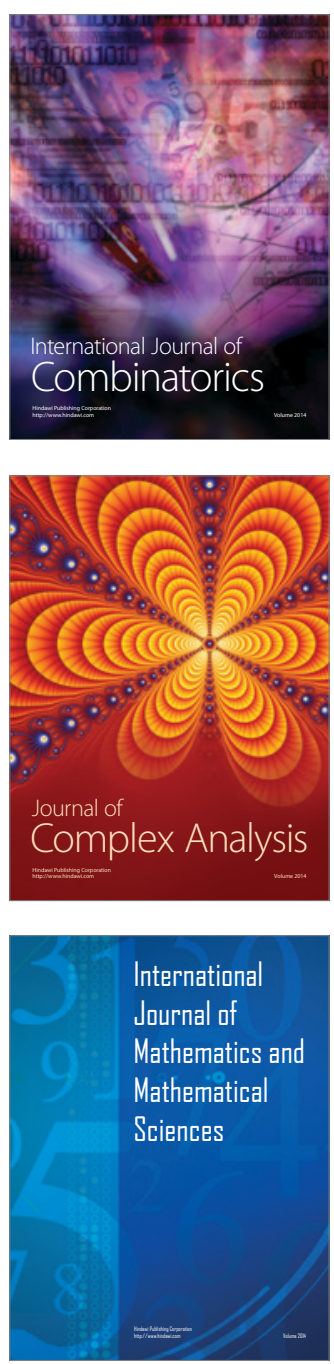
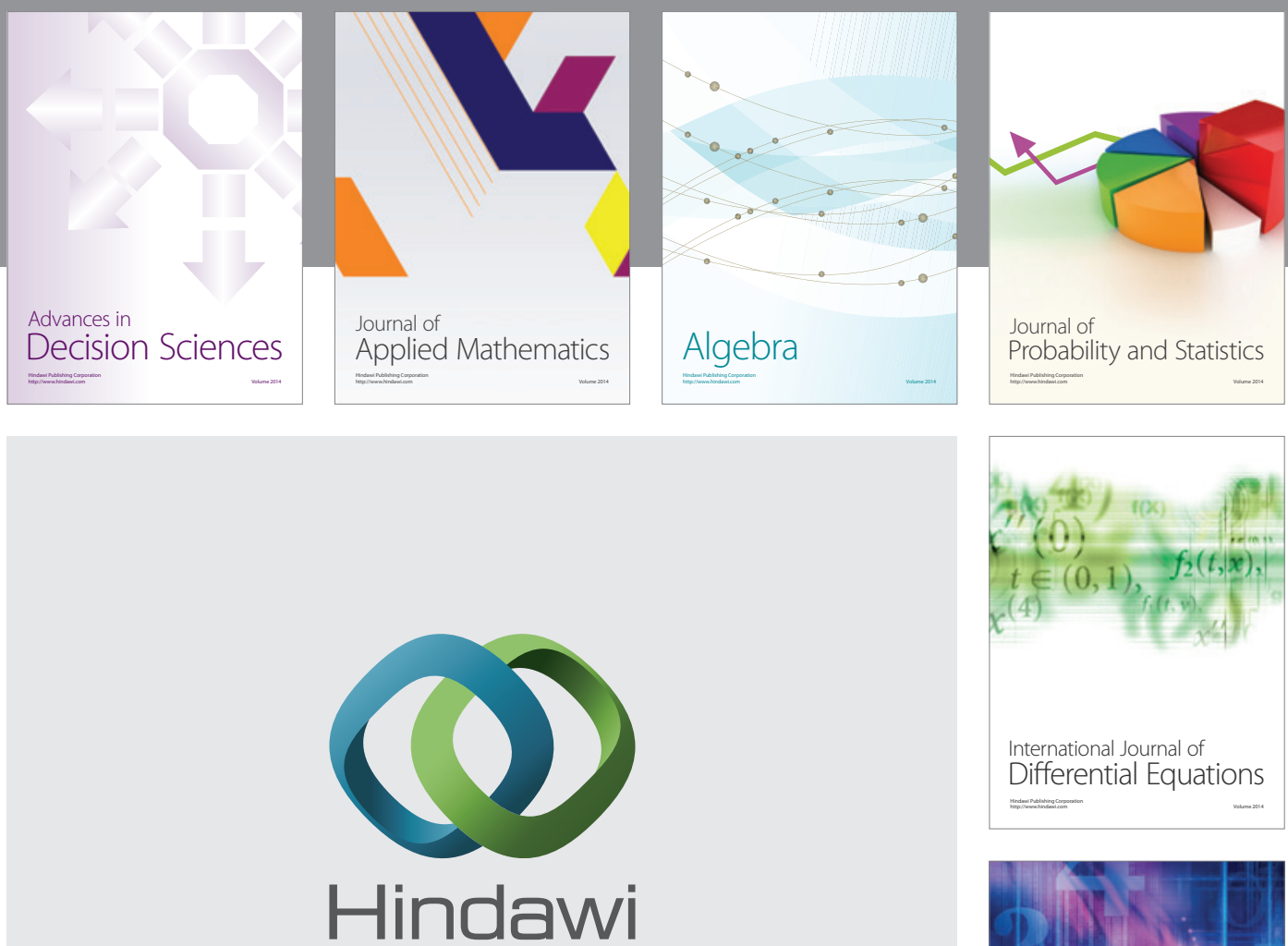

Submit your manuscripts at http://www.hindawi.com
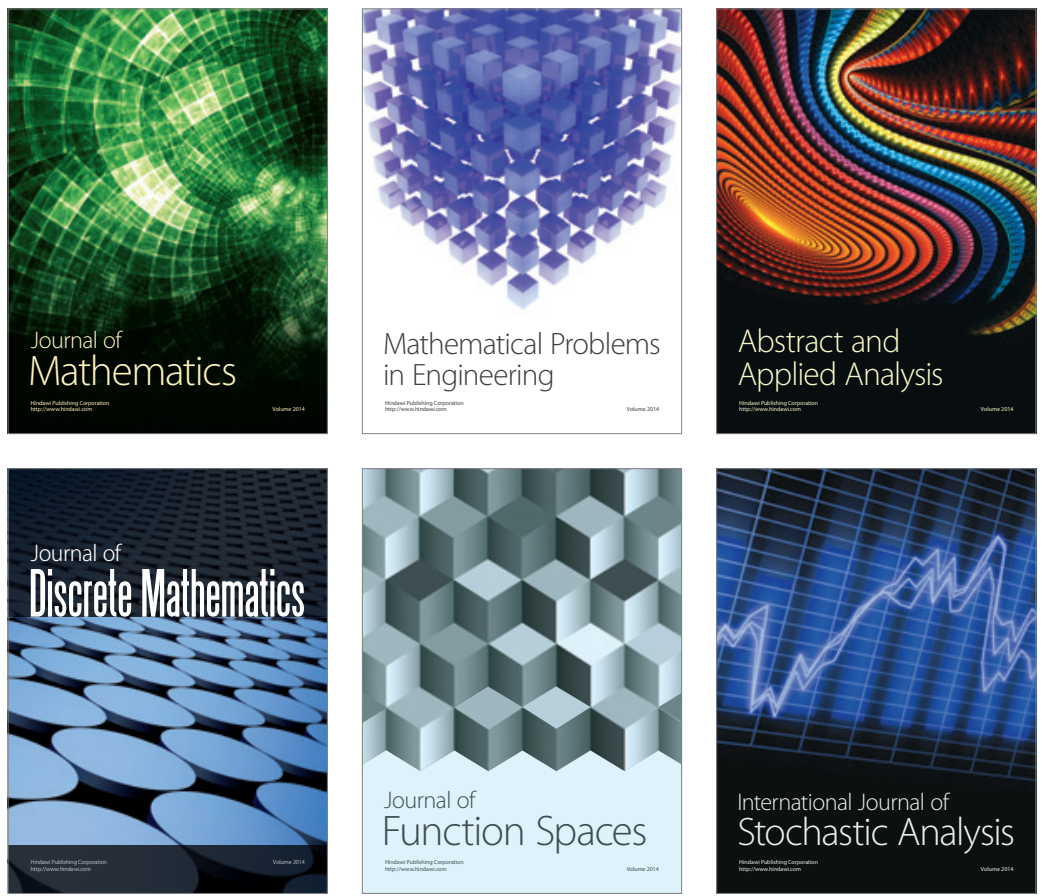

Journal of

Function Spaces

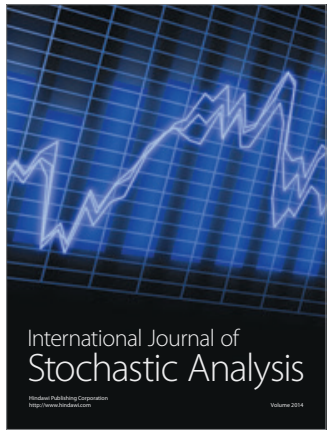

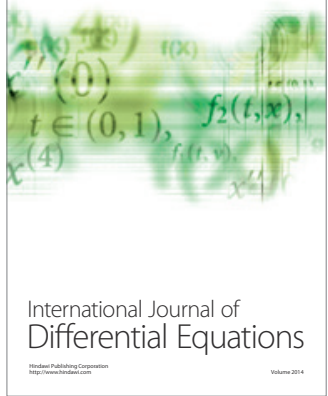
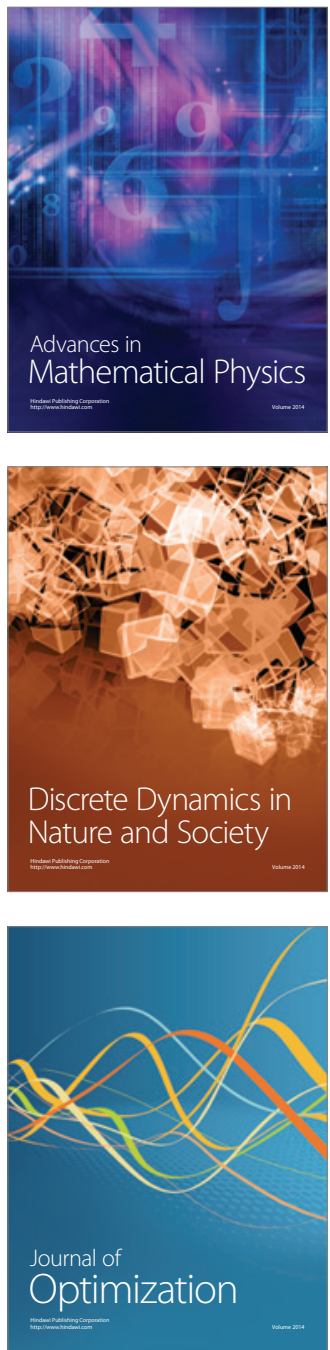